\title{
Bemerkungen über die Citronsäure und
}

\section{Gallussäure;}

\author{
von J. Robiquet.
}

Am Schlusse meiner Bemerkungen*) über die verschiedenen Modifikationen, welche die Citronsäure durch die Hitze erleidel, habe ich von der sonderbaren und schnellen Einwirkung der Scliwefolsünre euf dieselbe gesprochen. Ich habe diese Einwirkung studirt und obgleich ich nichts gefunden habe, was meine vorgefafste Meinung bestātigen konnte, will ich doch die Hauptsache davon mittheilen.

Die niedrige Temperatur, wobei die Einwirkung statt findet, war mir besonders aufgefallen; $25^{\circ}-30^{\circ}$ reichen hin, wenn beide Siuren nur die zu ihrem Bestehen ü̈thige Menge Wasuer enthalten. Wenn man 1 Th. trockene, gepulverte Citronsĩure mit 12 'Th. concentrirter Sclıwefelsë ure zusammenbringt, so löst sich Erstere leicht nach einigen Augeublicken und ohne die geringste Färbung; erhitzt man aber bis zu der angexeigten Temperatur, so bemerkt man bald eine Art innerlicher Bewegung, einer Gährung ahnlich, welche sich durch eine Menge feiner, allenthalben aufsteigender Gasblasen zu orkennen tibt, die sich mit einer bemerkenswerthen Regelmä fsigkeit entwickeln. Diese Gasblasen sind in Anfang fast reines Kohlenoxyigas. His ist wahrscheinlich, dafs es ein wenig Kohlenwasserstoffges enthïlt, deun zu seiner Verbrennung hedarf es immer einer etwas gröfseren Menge Sauerstoff, als dio in der dadurch erzeugten Kollensäure enthaltene heträgt. Auch besitzt dieses Ges gewöhulich einen schwachen kneblauchartigen Geruch, der fast verschwindet, wenn die Citronsiure ganx rein ist. Nach Verlauf einiger Stunden wird die Gas-

-) Aunal. do Pharm. Bd. XXXV. s. 138. 
entwickeiung schwacher und würde ganz aufhörex, wenn man die Temperatnr nicht um einige Grade erhöhte. Das KohJenoxydgas erscheint rein, bis die Temperatur ungefahr $40^{\circ}$ erreicht hat und dann erst erscheint, zuerst in sebr geringer Menge Kohlensïure, die sich aber mit der fortschreitenden Temperatur vermehrt, wo dafs bei $7^{\circ}$ das Knhlenoxydgas $b^{3} u z l i c h$ verschwunden ist und ervt bei $100^{\circ}$ zeigen sich Spuven von schwefliger Săure. Diese Binwirkung, welche ich 8 Tage lang, jeden Tag die Temperatur um angefahr $5^{\circ}$ erhöhend, unterhalten habe, hat is dem Gemische nur eine leichte uitrongelbe Färbung horrorgebracht, and man bemerkte in den Galeitungsröhren nicht die geringete Menge von Feuchtigkeit. Wenn ich unmittelbar bel $100^{\circ}$ operirte, bis eich kein Gas mehr entwickeite, was 5 bis 6 Stunden dunert, war der VerJwt, welohen die bel $100^{\circ}$ getrockncte Citronsarure erlitt, nie geringer als $53-55 \mathrm{pCt}$.

Wenn man durch Kalk oder Bargt den Räcketand sättigt, lot man ûber die geringe Menge organischer Materie erstannt, weiche dabei bleibt. Das unläsliche schwefelsaure Salz, welches entsteht, ist kanm grea gefirbt, wenn es im bedeckten Tiogel calcinirt worden ist und enthät nur Spurea ron Schwerelmetull. Die Waschfiasigkeiten hinterinsen beim Abdanpfen nur einen fost unwägbren Rtickstand. Wenn man indessen mit gröfseren Mengen arbeltet und den Rückstand durch kohlensanres Natron ätigt, so sicht man, wenn dis Operalion weit genug getrieben worden war, einige braune, harzähnliche, in Wasser unlösliche Klümpchen niederfallon, die oich in Alkohol und alkalischen Flüsolgkelten mit sehñner resenrother Farbe lösen. Es ist diefo eine rärbende Materio, welche keine Beständigkcit hat, und ven der wahrecheintich die röthliche Farbe herrihrt, wolche die ganze Fiüssigkeit besitzt. Sobald man dem Gemenge der beiden sturen Wasser sugenetxt hat, entwickelt sich ein dentlicher Geruoh nahh 
Essigsëure oder vielmohr Aceton, obglaich man keine Spur davon in dem Product der Bestillation weder vor, noch nach der Sätigung durch kohlensaures Yatron entdecken kann. Man erhält beim Abdampfen dieser genättigten Lösung, eine Erofse Menge schwefelenure Natron von schwach röthlicher Forbe der Mntterlauge. Man danpft von neuem ab bis sur Kryatallisation and fahrt so fort, bis man keine Krystalle melir erhält. Zuletzt bleibt eino sehr dunkel gofürbte Flüssigkeih wolche man mit Wasser verdiumt und durch essigseures Blei fallt. Der gewaschene Niedersehlag wird in Wasser zertheilt und durch einen Strom von Sebwefelwasserstofigas zersetzt; hierauf erhitzt man, filtrirt und dampft unlar der Luftpimpe ab. Das Product ist braun, klebrig, unkrystallisirbar und ziemlich suuer, wird weder von Baryt, Strontian, noch Kalk gefallt nnd seine Verbindungen mit diesen Bssen sclseinen uskryatallisirbar zu seyn. Diese Sâre, welche man nur in soringer Menge erhālt und die aufserdem nur negative Eigenschaften zu besitsen scheint, hat zu wenig lateresse dargeboten, um sie genauer $\mathrm{zu}$ studiren; ich beschrïnke wich dar. auf, thre Existena anzuzeigen.

Was mich veranlofet hat, auf diesen Versuch surücksnkommen, war das Verlangen an erfahren, was aus der Citronsăure nach dteser Abgabe von Kohlenoxyd würde, wan in diesem Falle, wie bei der blofsen Einwirkung der Ilitse, sich von Anfang der Operation an entwickelt, mit dem einzigen Unterechiede, dafo durch die Sshwefelsäure, dle Modification bei einer viel niedrigern Temperatur erfolgt, wodurch ich eie viel boetianter in erhalten hofite. Es iat mir jedoch bel allor Veralnderung des Verhältnisses und Concentrationggrades der Säure, nic gelungen, die Reaction auf die blofe Entwick-. lung des Kohlenorydgases zu beschränken, was ohne Zweifel daher rübrt, dafs das Product der ersten thinwirkung selbst vieder verïndert wird und die Entwickeleng ron Kohleusiavice 
bedingt. Wie dem auch sey, on findet die grófste Analogie in den Veräuderungen statt, welche die Citronsäure einerseits durch Schwefelsäure bei geringer Temperaturerhöhung, andorerseits durch die Hitze allein erleidet. In beiden Fällen erzeugt sich Kohlenoxydgas, Koblensïnre, Wasser und Aceton; denn ich glaube nicht, dafis man die Bildung von Wasser durch den Einflufs der Sohwefelsture bestreiten knnn. Sie scheint mir hinlänglich bewiesen durch die geringe Monge organischer Materic, welche nuch Ausscheidung der Fase zarïckbleibt. Was das Aceton betrifft, so offentart es sich durch den Geruch. Es ist sehr wahrscheiulich, dafis die Frzengnng des Wassers Hauptsache ist und dafs die äbrigen Producte nur dic Folgen davon sind. Daraus erklärt es sich auch, dafs diece secundären Producte baid in gröfserer bald in geringerer Menge auftreten und vicht blofs Kohlensäure sind, wle man behauptet hat.

Als ich die Resultate der Einwirkng der Ilitze anf dio Citronäure beschrieb, habe ich unter den zahlreichen Producten, welche sich dabei bilden, ein mit blauer Flamne brennbares Gas angeführt, von dem ich sagte, es sey Kohlenoxydgas. Indessen sagt Liebig in seiner Abhandiung über die Constitution der organischen Säuren, wo er von der Citrongäure spricht, dafs sie kein brenubares (ies liefere, weun man sit der Kinwirkung der Ilitze aussetze.*) Diesc Behanptung ist um so auffallender, uls es sich nur un eine Thatsache handeit, über die keine Tänzchung möglich ist, und die nicht von dem Untersehiede der Temperatur herrühren kann; denn ich habe genan angezeigt, dafs dieses Ges sich bei $160^{\circ}$ und ein wenig darüber entwickelte. Es ist indessen gerifs, dafs, wenu man

- Ion habe vicht die entfernteste Absieht gehabt, das Anftreten des Kohlenuxydgases bei dieser Destillation za bestätigen oder zu widerlegev, und habe noch viel weniger daran gedacht, dle Beobachtuig des Hra. Robiquet in Zweifel 23 ziohan.

J. $\mathbf{L}$. 
2u uchnell erhitzt, sich sogleich weh Kohlensinure in so grosscr Menge bidet, dafs sie, wenn man sie aicht durch eine alkalische Fhissigkeit absorbiren läfat, - die Eatflammung des Kohlenorydguses hindert. Wis ist anch begreiflich, dafo, da die Menge des Kohlenoxydgases ziemlich unbedeutend ist im Vorhältuils zur gunzen Menge der Citronsāure, besonders wenn man die Süare in einzelnen Krystallen anwendet, dieses Gas oehr wohl unbemerkt bleiben kann, wean man nur mit einer geringen Menge arbeitet. Es verhält sich danit, wie mit dem Aceton, welches sugleich auftritt, denn es gelingt nur, wenu men mit ziemlich grofsen Mengen von Citronsäure arbeitet, einige Grammen davon zu erhalten. Man kenut übrigena eine Menge Beispiele dieser Art. Ich weifs, dafs man immer eher sich selbst traut, als Andern, wena es sich aber um eine Thatsache mit so anfallenden bigenschaften haudelt, worüber kein Irrthum obwalten kann, begreife ich nicht, dafo man ohne Rückhalt nbspricht, was ein Andrer bestätigt hat. Wenn W ittetock sagt, dafs die Morphiusalze iurch Gerbestoff nicht gefillt werden, so glaube ich es, weil ich es nicht für möglich halte, dafs er sich in dieser Bexiehung irren konute. Wenn dagegen Hr. Pelouze behauptet, dafs sie gefaillt werden, glaube ich es ebenfolls nnd immer aug demselben Grundo and ich ochreibe diese Abweichung besondern Uıutänden zu, die von dem Einen oder dem Audern nicht genugsam berücksichtigt wurden. Ich mufs gestehen, dals jch un so woniger wufte, was ich davon halten sollte, als alie Morphinsalze, welche ich gepriift hatte, durch den Gerbstoff nicht gefält wurden. Nachdem ich aber, gemeinschafilich mit Hru. Bussy, ein Produckt dieser Art zu studiren Gelegenheit gehabt und gefunden hatte, dafs iurch dasselbe Agens einmal Fallung eintrat, ein andermal nicht, befunden wir uns auf diese Weise in der Nothwendigkeit, die Sache näher zu untersuchen, nod erkannten bald, dafs diese Anomalie ron dem Zustonde 
ast Robiquet, Bemerkungenüb.d. Citroustiure n. Gallussäure.

der Saturation des Selzes abhing and dafs die Fullung nur bel voltkommner Neutralität stettfand. Diese Bcobahtung veranlafste uns, uns zu überzeugen, ob nicht dureelbe Fall auch bei den Morphinsalzen stattinde und wir fanden in der That, dafs das salzsaure Salz, welches sich bei der Prüfung mit Lakmuspapier als neutml erwies, demungeachtet aber durch Gerbstoff nicht gefallt wurde, diese Eigenschaft uugenblieklich erlangte, wenn man einen Tropfen eufserst schwachen Ammoniaks zurügte. Die dazu nöthige Menge wax so gering, dafs es hinreichte, der Oberfläche der Auftösung eine mit Ammoniak befeuchtete Röhre zu nähern, um augenblicklich einen weifuen käsigen, dem Chlorsilber ähnlichen Niederachlug entstehen su sehen.

Ich hatte auch in meiner Abhandlung über die Citronsă zre gcsagt, dafs es mir gelungen sey, die Citronsäure von Ba up darzustelten; ich habe selbst die sehr einfache Bereitungsurt angegeben, welche durin bestehı, dafs man die Pyrocitronsäure lange Zeit in einem Destillirapparat auf $130^{\circ}$ erhitat. Fast die ganze Menge der Pyrocitrousäure geht ïber, wihrend die Citronsüare krystalliuisch surückbleibt, und eo ist leicht, sie durch blofue Aufiösung und Krystallisation zu reinigen. Ich habe die Analyse nicht wicderholt, aber alle charucteristioche Merkmale an ihr gefunden, dio Herr Buvp angibt and besitse davon noch vielleicht 00 Grammen. Ihr Geschmack, ihre Löslichkeit, ihre Krystallform sind so verschieden, dafs man sie nicht verwechseln kann.

Was die seit langer Zeit durch Dahlströhm angeaelgte Säure betrifft, so hätte ich ebenfalls gewìuscht, sic mir zu verschaffen, meine Versuche waren jedoch vergeblich, was ohne Zweifel daher rührt, dafs wir darüber zu wenig Mitthellangen besitzen. Sa sagt Berzelius blos von ihr, dafs sie entotehe, wenn man die Citronsäure bei ungefähr $200^{\circ}$ behandle und er figt hinzu, dals sie eine um so stärkere Mo- 
difieation orleide, als man die Hitze linger darauf elnwirken lasoe. Dabletröm sagt, dafs diese neuo Sïure in Aether 16̆blich sey und ieh gleubte darin ein vortreffliches Mittel, sio auszuniehen, zn inden. Latte indersen nicht den Erfolg, welchen ich davon erwartete, doch bat es mir Gelegenheit gegeben, eine ziemlich interessante Bemcrkung zu machen. Die Citronsäare sebmilzt, wie ich schon angeführt habe, bei $100^{\circ}$ und wenn men sie nach dem brkalten mit wasserfreiem Aether behandelt, lôst sie ich vollständig darin; es bedarf sogar dazu nur einer sehr geringen Menge Aethers, was ich mir aber nicht zu erklären weifs, ist, dafs wach einigen Stunden eine Menge von kieineu Krystallen niederfullen, die um so betrichtlicher ist, als dic Säure kürzere Zeit erhitzt worden war und, wenn man sich blos auf die zum Sehneelnen erforderliche Zeit beschrinkt hat, bleibt fast nichts in Aufiosung. Es ist zu bemerken, dafs alles diefs in vollkominen varschlossenen Gefifse stuttindet und ohne dafs man es einer Verduartung suschreiben könnte und, was noch auffallender ist, wenn diese Krystelle sich eiumal abgesetzt haben, sind sie nicht mehr merklich löslich in Aether. Ich glanbte anfinglich; defs diefs gerade die Saure von Dahlström mey, abor diese Iryatalle haben bei genauer Untersuchung alle Charactere der gewöhnlichen Citrousäure gezeigt. Mit kohJensaurem Natron gesättigt und durch nentrales salpetersaures Silberoxyd gefallt, lieferte das entstandene citronsaure Sllheroxyd bei der Analyse gena die durch J. G a y L nssac angezcigten Verhältnisse. Ich habe auch bei dem titronsaureu Silberoxyd die von J. Gay-Lussac und Liebig angezeigts Erscheinung des Verpufteus beobachtet und ex ist mir nur daderch zelungen, es zu vermeiden, daf's ich das Salz mit AlIrohol hefeachtete. The ich diese kurze Notiz schliefse, will ich noch über das galluseaure Ammoniak eine A uskunfi geben, con dem liebig in meiner Abhandlung spricht. Diever be- 
rühmte Chemiker sagh, daf́s er nicht wisse, wie das kryatallisirte gallussaure Ammoniak bereitet sey, von welchem er eine Probe von mir erhalten hat. Ich glaubte cs ihm mitgetheilt zu haben und lasse hier folgen, was ich im Juni 1896 darüber bekannt gemacht habe. "Ich habe gefunden, dafs, wean man unter gewissen Umständen diese beiden Körper (Ammoniak und Gallussäure) mit einander in Berührung bringt, eina wirkliche Verbindung und Bildung eines Salzes stattindet, welches in kaltem odcr heifsem Wasser gelöst werden kwn, krystallisirbar ist, Krystallwasser enthält und an der Luft keine Veränderung erleidet. Aber nur das saure gallussaure Salz rerhält sich so und wenn mau von wasserfreier Säure so vicl trocknes Ammoniakgas verschlucken läfst, als sie autiehmen kana, so erhilt man immer ein basisches Salz, Relust wenn man es lange Zeit im luftleeren Raume stehen lïfst, um alte onhängende Ammoniak zu encfernen. Wis reicht wicht hin, den Uebersehufs an Base zu sättigen, um ein unveränderliches Salz su erhalten, man mufo durchaus die Nenge dor Säure verdoppeln, d. h. ein Bigallat bilden. Auf diese Weise war dus bereitet, was Hr. Liebig analyeirt liat."

Hr. Liebig zieht, bei der Elitwickelung seiner thenretischen Ansichten über die Pyrogallussĩure, die Constitution derselben, so wie sie durch die neuesten Arbeiten festgestallt worlen ist, in Zweifel und hält es fïr möglich, dafs die Gallussäuro der Eiıwirkung der Hitze ausgesetzt, eich nicht blos in Pyrogallussäure und Kohlensäure zersetzt. Er nimmt sofar an, dafs 4 Atome Gallussäure sich zersetzen in swei Atome Pyrogallussäure, ein Atom Metagalluseäure und 4 Atome Kohlensäure. Ich will hei dieser Gelegenheit daran erinnern, dufs ich in meiner Notiz über die Wirkung der Hitze auf die Gnllussäure*) ausdrücklich bestätigt habe, dafs ich bei dor Subli-

-) A uuqi. d. Pharm, Bd. XXV. S. 16L. 
mation der Galluseluure vic weniger alu $20 \mathrm{pCt}$. Rñckstand und niclit mehr ais $50 \mathrm{pCt}$. Pyrogallussäure erhalten konnte.

Endlich bleibt mir noch eine letzte Bemerkung über diese Stare zu machen übrig. IIr. Liebig macht in seiner Ablandlung darauf aufmerksam, dafs die Formel des Gerbstoffe sich anadräcken Järst durch zwei Atome Gallussäure und ein Atom Easigoünre und dufs hiernach seine Tersetzong durch den vertingerten Einflufs von Wasser und Luft sehr einfach scheint. Diesolbe Idee findet sich sehr deutlich in meiner ersten Notiz von Febr. 1837 augesprochen, icls setzle sogir hinzu, dafs ich mir vorgenommen hätte, diese Vermuthung durch den Vereuch zu bestätigen; aber leider werden nicht alle Schlüsue, die sich so einfach aus den Forneln ableiten lussen, durch die Resultate der Versuche gerechtfertigt, anch zieht man oft vor, und diefs ist das klügste, nicht eher Formeln aufzustellen, als nachdem die T'hatsachen gesprochen haben. Gewifs ist, dafs ich mir die gröfste Mühe gegeben habe, die Essigsure in der Fliissigkeit aufusuchen, welche iiber der in einem verachloneenen Gefărse aus Galläpfeln gebildeten Galluzsäure steht, ohne die geringate Spur davon zu finden. Ich hatte gleichfalla einer andern möglichen Umwandlung Erwähnung gethan, ohne sie durch das Experiment begtätigt zu finden. Man wird also wolll warten mïssen, bis andere Thatsachen oder Verwuthungen kommen, die Erklärung zu geben, die man schon gefunden zu haben glaubte und die entwischte. (Jourual de Pharm. Ferr. 18s9.) 